\title{
Predicting Adolescents' Self-Objectification from Sexualized Video Game and Instagram Use: A Longitudinal Study
}

\author{
Marika Skowronski $^{1}$ (D) Robert Busching $^{1} \cdot$ Barbara Krahé $^{1}$
}

Published online: 23 August 2020

(C) The Author(s) 2020

\begin{abstract}
A growing body of research has demonstrated negative effects of sexualization in the media on adolescents' body image, but longitudinal studies and research including interactive and social media are scarce. The current study explored the longitudinal associations of adolescents' use of sexualized video games (SVG) and sexualized Instagram images (SII) with body image concerns. Specifically, our study examined relations between adolescents' SVG and SII use and appearance comparisons, thinand muscular-ideal internalization, valuing appearance over competence, and body surveillance. A sample of 660 German adolescents ( 327 female, 333 male; $M_{\text {age }}=15.09$ years) participated in two waves with an interval of 6 months. A structural equation model showed that SVG and SII use at Time 1 predicted body surveillance indirectly via valuing appearance over competence at Time 2. Furthermore, SVG and SII use indirectly predicted both thin- and muscular-ideal internalization through appearance comparisons at Time 1 . In turn, thin-ideal internalization at Time 1 predicted body surveillance indirectly via valuing appearance over competence at Time 2 . The results indicate that sexualization in video games and on Instagram can play an important role in increasing body image concerns among adolescents. We discuss the findings with respect to objectification theory and the predictive value of including appearance comparisons in models explaining the relation between sexualized media and self-objectification.
\end{abstract}

Keywords Social media $\cdot$ Computer games $\cdot$ Sexualization $\cdot$ Body image $\cdot$ Self-objectification

There has been growing evidence that sexualization in traditional media may increase body image concerns for adults and adolescents (Ward 2016). However, media use among adolescents has profoundly changed in the past decades: Television, movies, and magazines have experienced a sharp decline, whereas video gaming and social media use are on a constant rise (Twenge et al. 2019). At the same time, longitudinal studies on the topic are rare (Vandenbosch and Eggermont 2015, 2016), with only few longitudinal studies including Instagram use and no known longitudinal studies including video games. In the current study, we seek to explore the longitudinal

Electronic supplementary material The online version of this article (https://doi.org/10.1007/s11199-020-01187-1) contains supplementary material, which is available to authorized users.

Marika Skowronski

skowronski@uni-potsdam.de

1 Department of Psychology, University of Potsdam, Potsdam, Karl-Liebknecht-Straße 24-25, D-14476 Potsdam, Germany associations of sexualization in these media types with male and female adolescents' body image concerns.

\section{Sexualization in Video Games and Instagram}

As defined by the American Psychological Association (APA), sexualization occurs when a person's value comes only from his or her sexual appeal while ignoring their personalities, when a person is treated as a sexual object, when sexuality is inappropriately imposed (e.g., in the case of children), and/or when a person's physical attractiveness is equated with his or her sexiness (APA Task Force on the Sexualization of Girls 2007). Sexualization is common in media popular among teenagers. In top-selling video games, characters are frequently depicted with sexually revealing clothing or partially nude (Downs and Smith 2010). Both male and female characters often feature unrealistic body proportions (Dill and Thill 2007; Lynch et al. 2016). These findings particularly pertain to female characters who are more likely than male avatars to appear sexualized (Downs and 
Smith 2010). Various content analyses have further documented frequent sexualization on social media (Carrotte et al. 2017; Davis 2018; Tiggemann and Zaccardo 2018). Many of the most popular influencers of 2019 are models, fitness coaches, bodybuilders, and fashion and beauty bloggers (Hopper 2019) who are likely to post sexualized images of their bodies (Liu and Suh 2017). Moreover, popular Instagram trends like "fitspiration" feature images that usually contain sexualized elements, like scantily-clad people with a heavy emphasis on appearance ideals (Ghaznavi and Taylor 2015; Tiggemann and Zaccardo 2018). As in video games, women are more likely than men to be depicted in a sexualized manner within fitspiration imagery (Carrotte et al. 2017), yet both women and men are frequently depicted wearing revealing outfits or no clothing at all (Deighton-Smith and Bell 2018).

In Germany, $64 \%$ of teenagers report using Instagram, which scores as the second most popular social medium after WhatsApp (Medienpädagogischer Forschungsverbund Südwest 2019). With adolescents spending almost $3 \mathrm{~h}$ per day on social media (DAK-Gesundheit 2017), they are likely to be exposed to a high number of images on a regular basis. In addition, $87 \%$ of teenagers report playing video games, with young women spending over $5 \mathrm{~h}$ and young men over $13 \mathrm{~h}$ per week gaming (Medienpädagogischer Forschungsverbund Südwest 2019). Taken together, these numbers draw a clear picture: The level of sexualized content is high on Instagram and in video games, and they are among the most popular media formats for current youth. Studies suggest that sexualization can have a negative impact on body image (Ward 2016). Given that adolescence is a particularly vulnerable phase for appearance-related social pressure (Helfert and Warschburger 2013), more research on the potentially adverse effects of sexualization in media popular among teenagers is needed.

Objectification theory (Fredrickson and Roberts 1997) proposes an explanation on how sexualization in the media might affect body image concerns. Following Fredrickson and Roberts (1997), women in Western societies learn from an early age that their body is looked at and evaluated by others (objectification). As a result, women are socialized to take an observers' perspective on themselves, valuing their body for its appearance and correspondence with society's current appearance ideal. This process is called self-objectification. There have been different approaches to operationalizing the construct of self-objectification because researchers understand it as a multidimensional concept (Moradi 2011). At the cognitive level, it is manifested by women's tendency to value appearance over competence (further referred to as valuing appearance); at the behavioral level, it is shown through persistent body surveillance (Calogero 2011). Both valuing appearance and body surveillance have been linked to a wide range of negative outcomes for women, such as eating disorders and depression (Peat and Muehlenkamp 2011; Tiggemann and Williams 2011). Although not explicitly designed as a media theory, self-objectification theory proposes that sexualization in the media constitutes one form of objectification and contributes to the development of selfobjectification (Fredrickson and Roberts 1997). In line with this reasoning, correlational research has found links between the use of sexualized media and self-objectification (Aubrey 2006; Morry and Staska 2001; Vandenbosch and Eggermont 2012). Experimental studies demonstrated that the exposure to sexualized media heightens self-objectification momentarily, drawing individuals' attention to their body in the respective situation (Karsay et al. 2017; Ward 2016).

Objectification theory was originally designed to describe the experiences of women in a culture that objectifies the female body. However, there is increasing evidence that the theory might also apply to men: Although men tend to have lower levels of self-objectification, data suggests that the underlying relations and patterns between media use and selfobjectification are similar (Moradi and Huang 2008; Vandenbosch and Eggermont 2015). Still, research is needed to examine similarities and differences between genders (Ward 2016). Furthermore, studies are missing that examine whether and how the use of new and currently popular media formats, like Instagram and video games, are linked to selfobjectification.

\section{Sexualized Media Use and Self-Objectification}

Evidence on links between video game use and selfobjectification is scarce, especially with adolescent samples. Vandenbosch et al. (2017) let adolescents play a video game with a sexualized or a non-sexualized character and found that playing with a sexualized avatar increased valuing appearance. Two more experiments supported this assumption (Fox et al. 2015), whereas a null effect was found by Read et al. (2018). To our knowledge, no single cross-sectional or longitudinal study on sexualized media and selfobjectification included video games in the design. Harrison and Bond (2007) measured male adolescents' consumption of gaming magazines and found that it predicted their drive for muscularity, a concept linked to self-objectification (Martins et al. 2007), measured 1 year later. Although Harrison and Bond did not measure actual video game use, this finding might be the result of their participants' exposure to video game characters in the magazines, which often emphasize the avatars' bodies and their sexual appeal (Dill and Thill 2007). The authors found this relation only for White, but not for Black adolescents, which could be explained by the lack of Black video game characters and therefore less identification possibilities for Black young men (Harrison and Bond 2007). Clearly, studies are missing that measure male and 
female adolescents' actual video game use and its associations with body image concerns in a longitudinal design to draw conclusions about the long-term outcomes of sexualized gameplay. To address this lacuna, the current two-wave study seeks to investigate whether sexualized video game (SVG) use leads to higher self-objectification.

Concerning social media use, research using the framework of objectification theory to connect it to body image concerns has increased greatly in the past decade (Holland and Tiggemann 2016). Many correlational studies demonstrated that higher Instagram use is linked with higher valuing appearance and body surveillance in women (Cohen et al. 2017; Fardouly et al. 2017; Feltman and Szymanski 2018; Saiphoo and Vahedi 2019). This correlational evidence is complemented by some experimental studies: Tiggemann and Zaccardo (2015) showed that after viewing fitspiration images compared with travel images, women scored higher on body dissatisfaction, a concept positively linked to selfobjectification (Tiggemann and Lynch 2001). In another experiment, female undergraduates reported greater body dissatisfaction after viewing thin- and athletic-ideal, but not muscular-ideal fitspiration images (Robinson et al. 2017). Research including men is largely missing. However, some longitudinal research has investigated the long-term associations of social media use with self-objectification for male and female adolescents: Sevic et al. (2019) studied social networking use and body image of male adolescents over 22 months and found no evidence of longitudinal relationships between social-media use and body surveillance. In another study by Vries et al. (2016), social-networking use predicted adolescents' body dissatisfaction 18 months later. Also, Wang et al. (2019) found that selfie-viewing predicted increased valuing appearance among adolescents 6 months later. However, all of these studies measured general social-networking use or selfie-viewing and did not specifically focus on sexualization. Only Vandenbosch and Eggermont (2016) used the extent of monitoring attractive peers on social media as a measure of sexualized social-networking use and found that it predicted valuing appearance and body surveillance among female and male teenagers 6 months later. However, studies are missing that measure adolescents' habitual consumption of sexualized Instagram images (SII) and its relation to self-objectification.

To sum up, there is only limited longitudinal research on media use and self-objectification (Aubrey 2006; Sevic et al. 2019; Vandenbosch and Eggermont 2015, 2016; Wang et al. 2019), with no more than two known studies including social media and no known study including video games. This is unfortunate because levels of sexualization are high on both media formats (Carrotte et al. 2017; Downs and Smith 2010), and they are among the most important media formats for current youth (Medienpädagogischer Forschungsverbund Südwest 2019). Moreover, video games and Instagram differ from traditional media by their interactive design, but the impact of interactivity remains unclear on the basis of past research. It has been argued that interactivity may create greater effects via a stronger feeling of presence (Karsay et al. 2017), but other research suggests that interactivity may diminish negative effects when cognitive load in video games is high (Read et al. 2018). Therefore, research on long-term relations of the use of sexualized video games and Instagram images with self-objectification is clearly warranted.

\section{Mediating Processes}

Fredrickson and Roberts (1997) argue that repeated exposure to objectification, as in sexualized media, gradually socializes women to self-objectify. It is likely that several mediating processes are involved in this process (Aubrey 2007). Two prominent variables are the internalization of appearance ideals and appearance comparisons, in general or with peers in particular (Fardouly et al. 2015), which are both argued to heighten self-objectification (Ward 2016). A prominent model featuring appearance-ideal internalization is the three-step model of self-objectification by Vandenbosch and Eggermont (2015). Following these researchers, sexualized media is proposed to increase valuing appearance directly and indirectly via appearance-ideal internalization. Both of these pathways then increase body surveillance as the third step. This model has been tested with adolescents' traditional media use and general Facebook use. However, studies are missing that apply the theory to the new media formats of Instagram and video games.

Concerning Instagram use, some cross-sectional evidence shows that Instagram use predicts self-objectification via appearance-ideal internalization and appearance-comparison tendencies in women (Fardouly et al. 2017; Feltman and Szymanski 2018). One cross-sectional study with men found that consuming fitspiration imagery on Instagram predicted body dissatisfaction through both muscular-ideal internalization and appearance comparisons (Fatt et al. 2019). These results suggest that both variables play a role in explaining the associations of sexualized media with self-objectification. Yet within the framework of objectification theory and the three-step model of self-objectification, no known study has included both mediators in longitudinal research on the role of sexualized Instagram and video game use. Hence, it is unclear if and how these variables mediate the relation between media and self-objectification.

Theoretical considerations may be derived from sociocultural theory (Thompson et al. 1999), which postulates that media use is one of three sociocultural predictors (in addition to peers and parents) of body image concerns. The model posits that appearance comparisons and appearance-ideal internalization are mediators of this relation, which has been supported by extensive research (Shroff and Thompson 
2006; van den Berg et al. 2002). Furthermore, researchers investigating the theory found evidence for a direct path from appearance comparisons to appearance-ideal internalization (Keery et al. 2004; Shroff and Thompson 2006). To our knowledge, no study so far has integrated this theoretical perspective into the three-step model of self-objectification. Applying these considerations to the framework of objectification theory, we expected sexualized media to increase appearance-ideal internalization directly and indirectly via appearance comparisons. Appearance-ideal internalization should then increase self-objectification.

\section{The Current Study}

We aimed to contribute to research on objectification theory in several ways. First, the three-step model of self-objectification (Vandenbosch and Eggermont 2015) is a prominent model to describe the associations of sexualized media use with body image concerns, but it needs further testing (Ward 2016). Although many studies have demonstrated links between sexualized media use and individual variables of the three-step model, few researchers have included all variables in their analyses. Second, although appearance comparisons are discussed as a further mediator in this process (Ward 2016), research integrating this variable in the process of selfobjectification is limited. With the current study, we aimed to address this gap by including appearance comparisons, appearance-ideal internalization, valuing appearance, body surveillance, and sexualized media use in a longitudinal design.

Third, scholars have called for more research on media use and objectification theory among males (Ward 2016), which we addressed in this study by including both male and female adolescents. Western societies define a thin body as ideal for women and a muscular body as ideal for men (Moradi 2010). However, both concepts are linked because muscular bodies are usually slender bodies as well (Schaefer et al. 2017). Furthermore, the current female ideal features both thinness and muscularity (Bozsik et al. 2018; Tiggemann and Zaccardo 2018). Based on this evidence, both appearance ideals should be relevant for male and female adolescents. We therefore included two facets of appearance-ideal internalization in our model: thin-ideal and muscular-ideal internalization. Because scholars have called for a more thorough examination of the concept of internalization (Karsay et al. 2017), this represents another aim of our study. Following the rationale of the three-step model of self-objectification (Vandenbosch and Eggermont 2015), sexualized media use should trigger valuing appearance over time, a claim that has been supported by several longitudinal studies including magazines, television, and specific social media behavior (Aubrey 2006;
Vandenbosch and Eggermont 2015, 2016; Wang et al. 2019), but not with respect to the exposure to sexualized images on Instagram or video games. Filling this research gap by measuring adolescents' sexualized Instagram and video game consumption over time was another goal of our study.

The following propositions were examined on our study: First, we postulated that higher levels of sexualized video game (SVG) and sexualized Instagram images (SII) use at Time 1 would predict greater valuing appearance at Time 2 (Hypothesis 1). Second, we expected SVG and SII use to predict greater appearance comparisons (Hypothesis 2). Third, we expected greater SVG and SII use to predict higher thin-ideal internalization, and muscular ideal internalization (Hypothesis 3). Fourth, we expected greater appearance comparisons to predict higher thin-ideal and muscular-ideal internalization (Hypothesis 4). Because we only had two data waves, the associations predicted in Hypotheses 2 to 4 had to be modelled cross-sectionally at Time 1.

Building on the rationale of the three-step model of selfobjectification, we assumed that thin-ideal and muscularideal internalization at Time 1 would predict higher valuing appearance and body surveillance at Time 2 (Hypothesis 5). In line with Vandenbosch and Eggermont (2015), we further expected a direct path from valuing appearance to body surveillance (Hypothesis 6). Again, due to the two-wave design of our study, this association had to be modelled cross-sectionally at Time 2 . Furthermore, we assumed SVG and SII use at Time 1 to be indirectly linked to body surveillance at Time 2 via appearance comparisons at Time 1, thin-ideal internalization at Time 1 , and valuing appearance at Time 2 (Hypothesis 7). We expected the same indirect path with muscular- as for thin-ideal internalization at Time 1. Furthermore, we assumed indirect paths from SVG and SII use at Time 1 to body surveillance via valuing appearance at Time 2 (Hypothesis 8). Finally, we proposed indirect paths from SVG and SII use at Time 1 to body surveillance and valuing appearance at Time 2 via thinideal or muscular-ideal internalization at Time 1 (Hypothesis 9). Our proposed model reflecting these hypotheses is displayed in Fig. 1.

As control variables, we included age and body-mass index (BMI) because higher scores on these variables have been linked to differences in the variables of the selfobjectification process (Tiggemann and Lynch 2001). Furthermore, there are several indications that objectification theory is generally applicable to males, but that genderspecific associations might exist (Moradi and Huang 2008). For example, muscular-ideal internalization might have a stronger influence on men than on women (Moradi 2010). Accordingly, we explored the possible moderating role of gender. 
H1

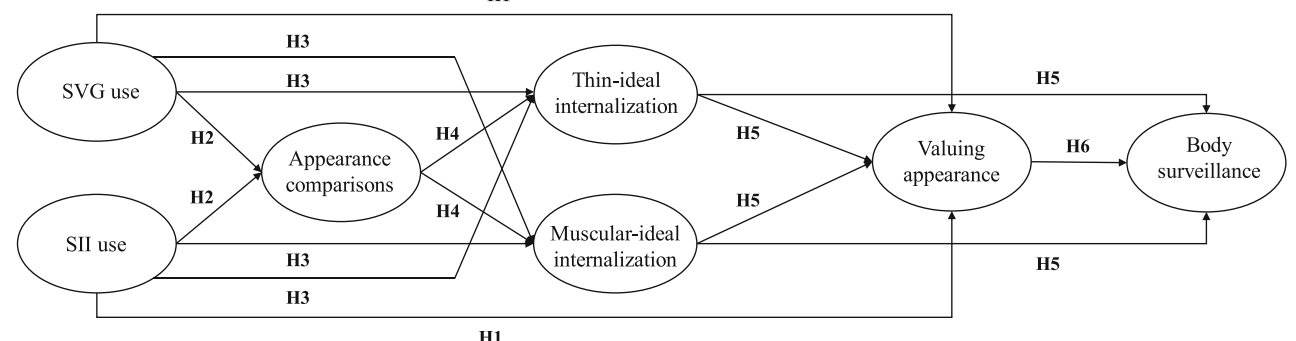

Fig. 1 Proposed model of the relations among SVG (sexualized video games) and SII (sexualized Instagram images) use, appearance comparisons, thin- and muscular-ideal internalization, valuing

\section{Method}

\section{Participants}

The sample consisted of 660 students ( 327 female and 333 male adolescents) from five secondary schools in different parts of Germany. The data were collected in two waves with an interval of 6 months. Of the 625 participants at Time 1 ( 316 female and 307 male), 544 took part at Time 2 (274 female and 270 male), resulting in a dropout rate of $12.96 \%$. Thirty-five participants $(5.30 \%$ of the whole sample) were absent at Time 1 and took part only at Time 2 . All 660 participants who participated in at least one data wave were included in the analyses, and missing data were handled using full-information maximum likelihood (FIML) estimation (see the following Analysis Plan section). The mean age of the sample was 15.09 years $(S D=1.26$, range $=$ $13-19)$ at Time 1 and 15.53 years $(S D=1.29$, range $=13-$ $19)$ at Time 2 . The majority of participants $(601,91.1 \%)$ were German nationals.

\section{Procedure}

Approval of the study was obtained by the Ethics Committee of the authors' university. The measures were administered by the first author during normal class hours. Informed consent was obtained from the participants themselves in line with the regulations for school-based research in the respective school administrations. Participants were told that the topic of the study was "Media use and self-perceptions." After providing informed consent, participants filled out the measures of body image concerns first and measures of media use second. At each data wave, participants could take part in a raffle of gift cards worth 10 or 25 Euros as a reward for participation. To ensure anonymity, participants created a personal code which was used to match the data for both time points. After the completion of the study at Time 2 , the researcher debriefed all participants during the rest of the class hour and answered questions about the study. Additionally, all participants were appearance, and body surveillance across time (T1-T2 is 6 months) as well as their relevant hypotheses $\mathrm{H} 1-\mathrm{H} 6)$. Indirect paths proposed in hypotheses 7 to 9 are not shown in the model

given a debriefing letter that contained contact data in case they had further questions.

\section{Measures}

\section{Use of Sexualized Video Games (SVG Use)}

SVG use was measured at Time 1. Participants were asked to list their two favorite video games that they had enjoyed playing most in the past 6 months. For each game, participants were asked to make two ratings: how sexualized (a) the female characters and (b) the male characters were on a scale from 1 (not at all) to 4 (very much). Examples were given for the meaning of sexualization for female and male characters each (e.g., scantily dressed for both, big breasts for females, muscularity for males). Participants were instructed to indicate " 1 " if there were no human-like female or male characters present in the game. The mean rating of the four items served as a measure of SVG use, with higher ratings indicating higher SVG use. The internal consistency was acceptable (Cronbach's $\alpha=.65$ ) bearing in mind the low number of items (Cortina 1993). The listing of favorite video games with content ratings by participants is widely used in the field of violent video game research as a reliable and valid method (Busching et al. 2015).

\section{Use of Sexualized Instagram Images (SII Use)}

SII use was also measured at Time 1. Participants were shown three pictures of sexualized women and three pictures of sexualized men taken from public Instagram profiles. The pictures showed young adults in various forms of scant clothing and body-emphasizing poses. For each male and female set, one picture reflected photos that are common among everyday users of Instagram (e.g., poses in front of a mirror/at the beach), one picture reflected sexualization in the context of fitness accounts, and one picture showed a person in an erotic pose, as commonly used for advertisements. Pictures are available from the first author upon request. For each picture, 
participants were asked to rate how often they had seen similar pictures on Instagram on a 5-point scale from 1 (never) to 5 (very often). Scores were averaged across items, with higher scores indicating higher SII use. Participants were told that they should not focus on the specific persons but the way in which they were presented (e.g., clothing, pose). Internal consistency was high (Cronbach's $\alpha=.93$ ).

\section{Appearance Comparisons}

The Physical Appearance Comparison Scale (Thompson et al. 1991) was used at Time 1 to assess participants' tendency to compare their appearance with the appearance of others in social situations. The scale consisted of five items (e.g., "In social situations, I sometimes compare my figure to the figures of other people") rated on a 5-point scale from 1 (never) to 5 (always). Scores were averaged across items such that a higher overall score indicated a stronger tendency to engage in appearance comparisons. Internal consistency for the scale was good (Cronbach's $\alpha=.83$ ).

\section{Thin-Ideal Internalization}

Thin-ideal internalization was measured at Time 1 with the Thin/Low Body Fat subscale of the Sociocultural Attitudes towards Appearance Questionnaire-4R (SATAQ-4R; Schaefer et al. 2017). The scale consists of three items (e.g., "I want my body to be very thin"), and participants rated the extent to which they strive toward appearance ideals on a scale from 1 (strongly disagree) to 5 (strongly agree). The mean rating of all items determined the participants' thin-ideal internalization score, with higher scores indicating higher levels of thin-ideal internalization. Internal consistency was good (Cronbach's $\alpha=.82$ ).

\section{Muscular-Ideal Internalization}

The Muscular/Athletic subscale of the SATAQ-4R was used at Time 1 (Schaefer et al. 2017). It consists of four items (e.g., "It is important for me to look muscular"). Participants rated the extent to which they strive toward the muscular ideal on a scale from 1 (strongly disagree) to 5 (strongly agree). The mean rating across all items yielded the participants' muscular-ideal internalization score, with higher scores indicating higher levels of muscular-ideal internalization. The scale had excellent internal consistency (Cronbach's $\alpha=.94$ ).

\section{Valuing Appearance over Competence}

Valuing appearance was assessed by the Self-Objectification Questionnaire (Noll and Fredrickson 1998) at Time 1 and Time 2. Participants were asked to rank 10 body attributes from 0 (least important) to 9 (most important) for their individual bodily self-concept. Five of the attributes are appearance-based (e.g., weight, sex appeal) and five are competency-based (e.g. health, stamina). The difference between the sum of the ranks of the appearance-based attributes and the sum of the ranks of the competency-based attributes determined the participant's score of valuing appearance, with higher scores indicating higher levels of valuing appearance. The maximum score for the appearance-based attributed was 35 , the minimum score of the competency-based attributes was 10 and vice versa, yielding a theoretical range of -25 to 25 on this measure. Due to its rank-order format, internal consistency could not be calculated for this measure. The measure has been shown to have good construct validity (Noll and Fredrickson 1998).

\section{Body Surveillance}

A shortened version of the Surveillance subscale of the Objectified Body Consciousness Scale was used at Time 1 and Time 2 (Knauss et al. 2008; McKinley and Hyde 1996). The scale consisted of seven items (e.g., "During the day, I think about how I look many times"), rated on a scale from 1 (strongly disagree) to 4 (strongly agree). Scores were averaged across items such that higher ratings indicated higher levels of body surveillance. Internal consistency was good at both data waves (Time 1: Cronbach's $\alpha=.81$, Time 2: Cronbach's $\alpha=.82$ ).

\section{Control Variables}

At Time 1, participants indicated their age as well as their weight and height, which was used to calculate their BMI.

\section{Analysis Plan}

Analyses of measurement invariance (MI) and the test of the hypothesized model were carried out using Mplus 8.4. The hypothesized model was tested using multigroup structural equation modelling. For appearance comparisons and thinideal and muscular-ideal internalization, the respective items were used as indicators for the latent variables. Due to its rankorder format, valuing appearance was included as a manifest variable in the model. Concerning SVG use, mean scores of the four items of the sexualized video game characters were used as to create a single-indicator latent variable. This was done because ratings of female and male characters in the same game were highly correlated $(r \mathrm{~s} \geq .58)$ and thus led to the model being non-identified. Concerning SII use, the ratings of the six sexualized female and male images were used as indicators for the latent variable. We used three item parcels as indicators for the body surveillance scale. When a scale measures a unidimensional construct, which is the case in our model, parceling enhances scale communality and 
modelling efficiency (Little et al. 2013). As recommended by Little et al. (2013), we used correlational parceling.

Before the hypothesized model was tested, measurement invariance for group comparisons between male and female participants was tested with Mplus 8.4 (Hussey and Hughes 2020). This pretesting was done for all variables except SVG use and valuing appearance, for which MI was not applicable because they are single-indicator or manifest variables. If MI holds, then male and female participants may be assumed to have interpreted the items and underlying latent factors in the same way (van de Schoot et al. 2012). Configural, metric, intercept-only, and scalar invariance models were tested using the robust maximum likelihood (MLR) estimator (van de Schoot et al. 2012). To determine differences between the models, we used the Bayes Information Criterion (BIC), because some of the models were nonhierarchical. The smallest BIC value represents the best fitting model (Raftery 1995). If full metric invariance was not given, some of the parameters were allowed to vary across groups to establish partial metric MI (Vandenberg and Lance 2000). We further tested MI across the data waves for body surveillance. MI across time was not tested for valuing appearance because it is a manifest variable. We specified a model that constrained the factor loadings on body surveillance to be equal across time and used BIC to compare this model to a baseline model in which factor loadings where allowed to differ.

In our proposed model, we controlled for the Time 1 values of age and BMI by including these variables as predictors for all endogenous variables. Furthermore, the Time 2 variables were controlled for their baseline value by including their respective Time 1 value as a predictor of the Time 2 value. Indirect paths were tested using the Mplus MODEL INDIRECT option. To test the significance of the direct and indirect paths, we calculated $95 \%$ and $99 \%$ confidence intervals through 10,000 bias-corrected bootstrap replications. Because bootstrapping is not available using MLR estimation, the maximum likelihood (ML) estimator was used for these analyses. However, bootstrapping as a nonparametric approach does not rely on assumptions of normality. The bootstrapping approach has been shown superior to all other procedures for testing indirect paths (Hayes and Scharkow 2013).

\section{Missing Data}

All participants who took part in at least one of the two data waves were included in the analyses. Missing data were handled using the FIML estimator of Mplus. This method has been shown to be superior to traditional missing data methods, yielding unbiased and more efficient estimates and standard errors (Enders and Bandalos 2001; Schlomer et al. 2010).

\section{Model Fit}

Because the Chi-square test is sensitive to sample size and degrees of freedom, even minor differences between the observed and estimated covariance matrices result in model rejection (Hair et al. 2019). Therefore, we report the Chi-square tests for completeness, but used the comparative fit index (CFI), the root-mean square error of approximation (RMSEA) and the standardized root mean residual (SRMR) to evaluate model fit. CFI values higher than .95, RMSEA values lower than .05 , and SRMR values lower than .08 indicate acceptable model fit (Hu and Bentler 1998; SchermellehEngel et al. 2003).

\section{Results}

\section{Measurement Invariance, Descriptive Statistics, and Correlations}

We tested MI across gender. Metric invariance was established for all variables except SII use. For SII use, we could establish partial MI, with three items having stronger loadings on the latent factor for the female compared to the male participants. We also tested MI across time for body surveillance. The assumption of weak measurement invariance was tenable, and factor loadings were comparable across time. (The fit indices and model comparisons are presented in the online supplement, Table 1s.) Table 1 presents the descriptive statistics and range for all study variables. We calculated differences between male and female adolescents with SPSS 26, using $t$-tests instead of multivariate analysis of variance, which uses listwise deletion. An alpha level of $p<.006(.05 / 9)$ was used to correct for multiple comparisons. The test statistics for gender differences can be found in Table 1. Significant gender differences were found for SII use, with female adolescents reporting higher consumption compared with males. Furthermore, female adolescents scored higher than did males on appearance comparisons, thin-ideal internalization, valuing appearance, and body surveillance. Male participants reported higher muscular-ideal internalization than did female. No gender difference was found concerning SVG use.

Table 2 shows the zero-order correlations between all variables in the model, separately for male and female participants. SII use and body image concern variables were significantly correlated for both young men and women (except for thin-ideal internalization, which was uncorrelated with SII use for male adolescents). SVG use was correlated with appearance comparisons, valuing appearance, and body surveillance for male adolescents. For female adolescents, SVG use was significantly correlated with body surveillance at Time 1 . 
Table 1 Scale range and descriptive statistics for study variables by participants' gender

\begin{tabular}{|c|c|c|c|c|c|c|c|c|}
\hline Time Variable & $\begin{array}{l}N \\
\text { items }\end{array}$ & $\begin{array}{l}\text { Possible } \\
\text { Range }\end{array}$ & $\begin{array}{l}\text { Total } M \\
(S D)\end{array}$ & $\begin{array}{l}\text { Young Women } M \\
(S D)\end{array}$ & $\begin{array}{l}\text { Young Men } M \\
(S D)\end{array}$ & $\begin{array}{l}\text { Gender comparison } t \text { - } \\
\text { test }\end{array}$ & $p^{\mathrm{a}}$ & $\begin{array}{l}\text { Cohen's } \\
d\end{array}$ \\
\hline T1 SII use & 6 & $1-5$ & $2.59(1.03)$ & $2.80(1.03)$ & $2.37(1.00)$ & $t(621)=5.33$ & $<.001$ & .43 \\
\hline T1 SVG use & 4 & $1-4$ & $1.66(.62)$ & $1.67(.65)$ & $1.65(.60)$ & $t(394)=.20$ & .84 & .02 \\
\hline T1 Appearance comparison & 5 & $1-5$ & $2.33(.83)$ & $2.53(.83)$ & $2.12(.77)$ & $t(620)=6.51$ & $<.001$ & .52 \\
\hline T1 Thin-ideal internalization & 3 & $1-5$ & $2.53(1.01)$ & $2.93(.98)$ & $2.13(.88)$ & $t(622)=1.79$ & $<.001$ & .87 \\
\hline $\begin{array}{l}\text { T1 Muscular-ideal internali- } \\
\text { zation }\end{array}$ & 4 & $1-5$ & $2.62(1.14)$ & $1.94(.87)$ & $3.32(.95)$ & $t(621)=-18.91$ & $<.001$ & -1.52 \\
\hline T1 Valuing appearance & 10 & $-25-+25$ & $\begin{array}{l}-12.11 \\
\quad(10.12)\end{array}$ & $-10.42(10.58)$ & $-13.95(9.25)$ & $t(600)=4.37$ & $<.001$ & .36 \\
\hline T1 Body surveillance & 7 & $1-4$ & $2.58(.62)$ & $2.82(.56)$ & $2.33(.58)$ & $t(622)=10.58$ & $<.001$ & .85 \\
\hline T2 Valuing appearance & 10 & $-25-+25$ & $\begin{array}{r}-10.69 \\
(10.43)\end{array}$ & $-9.34(10.56)$ & $-12.00(10.16)$ & $t(569)=3.07$ & .002 & .26 \\
\hline T2 Body surveillance & 7 & $1-4$ & $2.55(.60)$ & $2.78(.56)$ & $2.33(.57)$ & $t(576)=9.50$ & $<.001$ & .79 \\
\hline
\end{tabular}

Note. $\mathrm{SSI}=$ sexualized Instagram images; $\mathrm{SVG}=$ sexualized video games; $\mathrm{T} 1=$ Time $1 ; \mathrm{T} 2=$ Time $2(6$ months after T1 $)$

${ }^{\mathrm{a}}$ Critical $p(.05 / 9)=.006$

\section{Hypothesis Testing}

In a first step, we examined if gender was a moderator of the proposed associations. Therefore, we first conducted a multigroup analysis for male and female adolescents in which the paths were constrained to be equal for both gender groups. This model yielded a good fit, $\chi^{2}(694, n=660)=1152.424$, $p<.001$ (CFI $=.951, \mathrm{RMSEA}=.045,90 \%$ CI $[.040, .049]$, SRMR = .066). In a next step, we estimated a model in which the paths were allowed to vary between male and female adolescents. Again, the model fit was good, $\chi^{2}(661, n=660)=$ $1101.723, p<.001(\mathrm{CFI}=.953, \mathrm{RMSEA}=.045,90 \% \mathrm{CI}$ $[.040, .050]$, SRMR $=.056)$. Finally, we compared both models, using the BIC as the comparison criterion. The constrained model $(\mathrm{BIC}=48,195.92)$ had a smaller BIC value than the unconstrained model $(\mathrm{BIC}=48,359.46 ; \Delta \mathrm{BIC}=$ $-163.54)$, indicating better fit for the constrained model. This means that gender did not moderate the hypothesized relations. Despite this result, we could not employ singlegroup modelling but adopted the constrained multiple group model as the final model because we only had partial MI for SII use, with three items having stronger loadings on the latent factor for female than for male adolescents. Figure 2 depicts the standardized path coefficients of the final model, with a fit of $\chi^{2}(694, n=660)=1152.424, p<.001 \quad(\mathrm{CFI}=.951$, RMSEA $=.045,90 \%$ CI $[.040, .049]$, SRMR =.066). (The Mplus code and output is provided in Online Resource 2 in the online supplement.)

Hypothesis 1, which predicted that higher levels of SVG $(b=3.31, \beta=.17,99 \%$ CI $[.06,6.64])$ and SII $(b=1.15$,

Table 2 Correlations among study variables by participants' gender

\begin{tabular}{|c|c|c|c|c|c|c|c|c|c|}
\hline \multirow[t]{2}{*}{ Time Variable } & \multicolumn{9}{|c|}{ Correlations } \\
\hline & 1 & 2 & 3 & 4 & 5 & 6 & 7 & 8 & 9 \\
\hline 1. T1 SII use & - & $.24^{*}$ & $.25^{* * * *}$ & $.13^{*}$ & $.20^{* * * *}$ & $.14^{*}$ & $.17^{* *}$ & $.15^{*}$ & $.16^{* *}$ \\
\hline 2. T1 SVG use & $.14^{*}$ & - & .17 & .10 & .05 & .09 & $.23 *$ & .09 & .11 \\
\hline 3. T1 Appearance comparison & $.26^{* * *}$ & $.17^{* *}$ & - & $.43^{* * *}$ & $.16^{* *}$ & $.36^{* * *}$ & $.67^{* * * *}$ & $.28^{* * *}$ & $.62^{* * *}$ \\
\hline 4. T1 Thin-ideal internalization & -.05 & .04 & $.23^{* * *}$ & - & $.15^{* *}$ & $.25^{* * *}$ & $.48^{* * *}$ & $.26^{* * *}$ & $.40^{* * * *}$ \\
\hline 5. T1 Muscular-ideal internalization & $.36^{* * *}$ & .10 & $.41^{* * * *}$ & $.15^{*}$ & - & .05 & .10 & -.03 & .06 \\
\hline 6. T1 Self-objectification & $.28^{* * * *}$ & $.13^{*}$ & $.28^{* * * *}$ & .11 & .40 & - & $.42^{* * * *}$ & $.64^{* * *}$ & $.36^{* * *}$ \\
\hline 7. T1 Body surveillance & $.33^{* * *}$ & $.15^{* *}$ & $.60^{* * * *}$ & $.24^{* * * *}$ & $.43^{* * *}$ & $.35^{* * *}$ & - & $.37^{* * *}$ & $.73^{* * *}$ \\
\hline 8. T2 Self-objectification & $.25^{* * *}$ & $.25^{* * *}$ & $.22^{* * * *}$ & $.20^{* *}$ & $.34^{* * * *}$ & $.55^{* * *}$ & $.31^{* * * *}$ & - & $.40^{* * *}$ \\
\hline 9. T2 Body surveillance & $.26^{* * *}$ & $.17^{* * *}$ & $.53^{* * *}$ & $.15^{*}$ & $.40^{* * *}$ & $.30^{* * *}$ & $.74^{* * *}$ & $.36^{* * *}$ & - \\
\hline
\end{tabular}

Note. $\mathrm{SSI}=$ sexualized Instagram images, $\mathrm{SVG}=$ sexualized video games; $\mathrm{T} 1=$ Time $1 ; \mathrm{T} 2=$ Time 2 (6 months after T1). Correlations for female adolescents are above the diagonal of the correlation matrix; for male adolescents, below

${ }^{*} p<.05 .{ }^{* *} p<.01 .{ }^{* * *} p<.001$ 


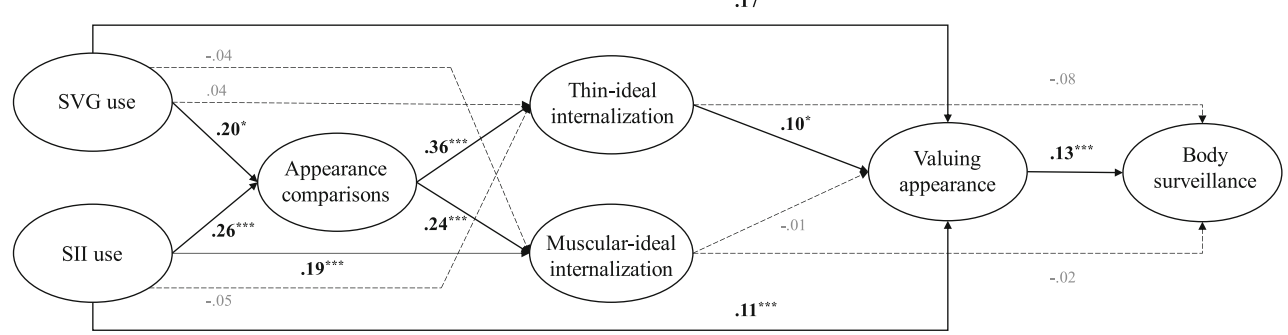

Fig. 2 Standardized path coefficients for the relations among SVG (sexualized video games) and SII (sexualized Instagram images) use, appearance comparisons, thin- and muscular-ideal internalization, valuing appearance and body surveillance across time (T1-T2 is 6 months).

$\beta=.11,99 \% \mathrm{CI}[.09,2.25])$ use at Time 1 would be linked to greater valuing appearance at Time 2 , was supported. The prediction in Hypothesis 2 that SVG $(b=.41, \beta=.20,95 \%$ CI $[.04, .75])$ and SII $(b=.26, \beta=.25,99 \%$ CI $[.11, .41])$ use would be associated with greater appearance comparisons was also confirmed. Hypothesis 3 stated that SVG and SII use would predict higher muscular-ideal and thin-ideal internalization. Partly consistent with this hypothesis, SII use $(b=.19$, $\beta=.19,99 \%$ CI $[.06, .32])$ was significantly associated with muscular-ideal internalization whereas SVG use $(b=-.04$, $\beta=-.02,95 \%$ CI $[-.36, .31])$ was not. Neither SVG $(b=.08, \beta=.04,95 \%$ CI $[-.27, .44])$ nor SII use $(b=-.05$, $\beta=-.05,95 \%$ CI $[-.16, .07])$ was linked to thin-ideal internalization, again not supporting Hypothesis 3.

Hypothesis 4, which predicted higher appearance comparisons to be associated with greater thin-ideal internalization $(b=.36, \beta=.36,99 \%$ CI $[.20, .53])$ and muscular-ideal internalization $(b=.24, \beta=.24,99 \%$ CI $[.11, .38])$, was confirmed. In Hypothesis 5, we assumed higher thin-ideal and
All analyses controlled for BMI and age. T2 variables controlled for the respective T1 values. Grey, dashed pathways are not significant. ${ }^{*} p<.05$. $p<.001$

muscular-ideal internalization at Time 1 would predict greater valuing appearance and body surveillance at Time 2 . Consistent with this hypothesis, thin-ideal internalization at Time 1 predicted valuing appearance at Time $2(b=.92$, $\beta=.10,95 \%$ CI $[.08,1.76])$, but we did not find this path for muscular-ideal internalization $(b=-.14, \beta=-.01,95 \%$ CI $[-.89, .65])$. In addition, the proposed paths from thin$(b=-.14, \beta=-.08,95 \%$ CI $[-.32, .03])$ and muscular-ideal internalization $(b=-.04, \beta=-.02,95 \% \mathrm{CI}[-.18,1.00])$ at Time 1 to body surveillance at Time 2 were not significant. Hypothesis 6, which stated that valuing appearance predicted body surveillance was confirmed $(b=.02, \beta=.13,99 \% \mathrm{CI}$ $[.004, .04])$.

The tests of the hypothesized indirect paths are presented in Table 3. A table displaying all indirect paths is presented in the online supplement, Table 2s. In Hypothesis 7, we expected SVG and SII use at Time 1 to be indirectly linked to body surveillance at Time 2 via appearance comparisons at Time 1, thin-ideal internalization at Time 1 , and valuing appearance at

Table 3 Indirect paths in the proposed model

\begin{tabular}{|c|c|}
\hline Indirect paths (Standardized) & $b[95$ or $99 \% \mathrm{CI}]$ \\
\hline SVG use T1 $\rightarrow$ Appearance comparisons $\mathrm{T} 1 \rightarrow$ Thin-ideal internalization $\mathrm{T} 1 \rightarrow$ Valuing appearance $\mathrm{T} 2 \rightarrow$ Body surveillance $\mathrm{T} 2$ & $.01^{*}[.003, .01]$ \\
\hline $\begin{array}{l}\text { SVG use T1 } \rightarrow \text { Appearance comparisons } \mathrm{T} 1 \rightarrow \text { Muscular-ideal internalization T1 } \rightarrow \text { Valuing appearance T2 } \rightarrow \text { Body surveillance } \\
\text { T2 }\end{array}$ & $.00[-.004, .001]$ \\
\hline SVG use T1 $\rightarrow$ Thin-ideal internalization T1 $\rightarrow$ Valuing appearance T2 $\rightarrow$ Body surveillance T2 & $.002[-.01, .02]$ \\
\hline SVG use T1 $\rightarrow$ Muscular-ideal internalization T1 $\rightarrow$ Valuing appearance T2 $\rightarrow$ Body surveillance T2 & $.00[-.002, .01]$ \\
\hline SVG use $\mathrm{T} 1 \rightarrow$ Valuing appearance $\mathrm{T} 2 \rightarrow$ Body surveillance $\mathrm{T} 2$ & $.08^{* *}[.02, .18]$ \\
\hline SII use T1 $\rightarrow$ Appearance comparisons T1 $\rightarrow$ Thin-ideal internalization T1 $\rightarrow$ Valuing appearance T2 $\rightarrow$ Body surveillance T2 & $.002[.00, .01]$ \\
\hline SII use $\mathrm{T} 1 \rightarrow$ Appearance comparisons $\mathrm{T} 1 \rightarrow$ Muscular-ideal internalization $\mathrm{T} 1 \rightarrow$ Valuing appearance $\mathrm{T} 2 \rightarrow$ Body surveillance $\mathrm{T} 2$ & $.00[-.002, .001]$ \\
\hline SII use T1 $\rightarrow$ Thin-ideal internalization T1 $\rightarrow$ Valuing appearance T2 $\rightarrow$ Body surveillance T2 & $-.001[-.01, .003]$ \\
\hline SII use T1 $\rightarrow$ Muscular-ideal internalization T1 $\rightarrow$ Valuing appearance T2 $\rightarrow$ Body surveillance T2 & $-.001[-.01, .003]$ \\
\hline SII use $\mathrm{T} 1 \rightarrow$ Valuing appearance $\mathrm{T} 2 \rightarrow$ Body surveillance $\mathrm{T} 2$ & $.03^{* *}[.003, .08]$ \\
\hline
\end{tabular}

Note. For nonsignificant results, the $95 \%$ CIs are presented.

${ }^{*} p<.05[95 \% \mathrm{CI}] .{ }^{* *} p<.01[99 \% \mathrm{CI}]$. 
Time 2The indirect path was statistically significant for SVG use $(b=.01, \beta=.001,99 \%$ CI $[.003, .01])$. However, for SII use, the indirect path was not statistically significant $(b=.002$, $\beta=.001,99 \%$ CI $[.00, .01])$. We expected the same indirect paths with muscular-ideal internalization at Time 1 , but they were not significant (SVG: $b=.00, \beta=.00,95 \%$ CI $[-.004$, $.001]$; SSI: $b=.00, \beta=.00,95 \%$ CI $[-.002, .001])$, because muscular-ideal internalization at Time 1 did not predict either valuing appearance or body surveillance at Time 2. Thus, Hypothesis 7 was partly supported for thin-ideal, but not for muscular-ideal internalization.

For Hypothesis 8, we expected an indirect path from SVG and SII use at Time 1 to body surveillance at Time 2 via valuing appearance at Time 2 . This hypothesis was confirmed (SVG: $b=.08, \beta=.02,99 \%$ CI $[.02, .05]$; SII: $b=.03$, $\beta=.02,99 \%$ CI $[.003, .08])$. In Hypothesis 9, we proposed indirect links from SVG and SII use at Time 1 to valuing appearance and body surveillance at Time 2 via thin-ideal or muscular-ideal internalization. These indirect paths were nonsignificant, not confirming Hypothesis 9 (for SVG use $\rightarrow$ thinideal: $b=.002, \beta=.00,95 \% \mathrm{CI}[-.01, .02]$ and SVG use $\rightarrow$ muscular-ideal: $b=.00, \beta=.00,95 \%$ CI [-.002, .01]; for SII use $\rightarrow$ thin-ideal: $b=-.001, \beta=-.001,95 \%$ CI $[-.01, .001]$ and SII muscular $\rightarrow$ thin-ideal: $b=-.001, \beta=-.001,95 \% \mathrm{CI}$ $[-.01, .003])$.

We conducted a sensitivity analysis to examine the influence of the covariates age and BMI on our model by specifying a model without controlling for these covariates. Apart from slightly different standardized coefficients, the results did not change, and the pattern of associations remained the same.

\section{Discussion}

The present study integrated theoretical considerations from objectification theory (Fredrickson and Roberts 1997) and sociocultural theory (Thompson et al. 1999) by demonstrating longitudinal associations between sexualized media use and adolescents' body image concerns. Specifically, our results show that sexualized video game (SVG) and sexualized Instagram images (SII) use indirectly increased body surveillance over time via several mediators: In the case of SVG use, via appearance comparisons, thin-ideal internalization, and valuing appearance, in the case of SII use, via valuing appearance.

We had several goals with the present study. First, we aimed to extend previous findings that sexualized media increase self-objectification over time, using valuing appearance and body surveillance as two components of selfobjectification and including Instagram and video games as media that are currently popular among youth. Consistent with our predictions and previous research concerning other media formats (Aubrey 2006; Vandenbosch and Eggermont 2015; Wang et al. 2019), adolescents' higher SVG and SII use at Time 1 predicted higher scores of valuing appearance at Time 2 . We also found that greater valuing of appearance predicted heightened body surveillance at Time 2 . The indirect path from SVG and SII use at Time 1 to body surveillance via valuing appearance at Time 2 was also significant. These results suggest that exposure to sexualized content on Instagram and in video games may negatively affect adolescents' body image. Our study is, to our knowledge, the first to examine longitudinal associations between sexualized images on Instagram, sexualization in video games, and different facets of self-objectification in male and female adolescents.

A second goal of our study was to shed light on processes mediating the path from sexualized media use to self-objectification. Appearance-ideal internalization and appearance comparisons have been discussed as mediators (Ward 2016), but to our knowledge, our study is the first to integrate both variables within a longitudinal model. In Vandenbosch and Eggermont's (2015) three-step model, sexualized media use predicts appearance-ideal internalization, which in turn predicted increased valuing appearance and body surveillance. However, the role of internalization is yet unclear because there are inconsistencies in the literature so that researchers have recommended a more thorough exploration of the concept (Karsay et al. 2017). For this reason and to reflect possible gendered manifestations of internalization, we included two separate measures of thin-ideal internalization and muscular-ideal internalization, respectively. Contrary to our expectations, which were derived from objectification theory and previous findings (Fardouly et al. 2017; Fredrickson and Roberts 1997; Vandenbosch and Eggermont 2015), we did not find associations of SVG and SII use with thin-ideal internalization at Time 1 in our model, nor did we find the proposed association of SVG use with muscular-ideal internalization at Time 1. Only the hypothesized association of SII use with muscular-ideal internalization at Time 1 was significant. Furthermore, we did not find the proposed paths from thinand muscular-ideal internalization at Time 1 to body surveillance at Time 2. In line with our hypothesis, we found that higher thin-ideal internalization at Time 1 predicted higher valuing appearance at Time 2 , but Time 1 muscular-ideal internalization did not. As a consequence, the hypothesized indirect paths from Time 1 sexualized media to Time 2 body surveillance via Time 1 thin-ideal or muscular-ideal internalization and Time 2 valuing appearance were also not significant.

In summary, neither thin-ideal nor muscular-ideal internalization mediated between sexualized media use and self-objectification, which is in apparent contradiction to previous research. However, there are several explanations for these results that might offer new insights into the concept of appearance-ideal internalization. Previous studies showing a 
mediating role of appearance-ideal internalization between sexualized media and self-objectification used the third version of the Sociocultural Attitudes towards Appearance Questionnaire (Thompson et al. 2004). In this version, appearance-ideal internalization was measured with items like "I compare my body to the bodies of people who appear in magazines" or "I wish I looked like the models in music videos."

This scale has several problems. First, it generally refers to the appearance of celebrities. Although celebrities usually represent current appearance ideals, this version does not explicitly capture the concept of thinness or muscularity. The fourth and revised version of the SATAQ used in the current study addressed this shortcoming by including subscales of thinideal and muscular-ideal internalization with statements like "I want my body to look very thin" or "It is important for me to look muscular" (Schaefer et al. 2017).

Second, the older version of the SATAQ does not measure internalization but rather the tendency of people to compare their appearance to others. In our model, this tendency is reflected in the variable "appearance comparisons," and as we expected, both SVG and SII use were linked to appearance comparisons at Time 1. In turn, appearance comparisons predicted thin-ideal and muscular-ideal internalization. Consequently, we argue that our study disentangled two processes involved in earlier operationalizations of internalization: (a) the tendency to compare one's appearance and (b) the internalized desire to have a body that looks a certain way - in our study either thin or muscular. Our data suggests that, when measured in this way, internalization might function differently from earlier operationalizations. Sexualized media might first trigger appearance comparisons that, in turn, promote the internalization of the thin ideal and the muscular ideal. Thin-ideal internalization then increases body surveillance indirectly via valuing appearance. The resulting full indirect chain from Time 1 SVG to Time 2 body surveillance via Time 1 appearance comparisons, Time 1 thin-ideal internalization, and Time 2 valuing appearance was significant, confirming our predictions. However, the same path was not significant for SII use. It might be that the indirect path is stronger for SVG use due to the higher presence of people experienced in video games. Following this rationale, the indirect SII use path might be explained by a lack of power for detecting a significant indirect path for the entire chain. Furthermore, no parallel pathway was found for muscularideal internalization, calling for future research to explore the role of muscular-ideal internalization.

Our findings have implications for objectification theory because they speak for the integration of appearance comparisons in the explanatory mechanism behind the associations of sexualized media with body image concerns. Highlighting further theoretical implications for objectification theory, the thin ideal seems to play a central role for the development of self-objectification, but the muscular ideal does not. However, we cannot conclude from this finding that muscular-ideal internalization is irrelevant for body image concerns because the concept is linked to body dissatisfaction and disordered eating (Fatt et al. 2019; Schaefer et al. 2017). Instead, the data offer some indication that the internalization of muscularity might either result in other forms of body image concerns or that other mediators between muscular-ideal internalization and self-objectification need to be considered.

The final goal of our study was to expand the literature on objectification theory by including male participants. In our investigation, we did not find gender to be a moderating factor of the examined associations. However, it is important to keep in mind that except for muscular-ideal internalization, female adolescents scored higher on all measures of body image concerns. Based on these data, the underlying processes are the same for male and female adolescents, but young women are still more inclined to be critical with their bodies, thus being more at risk for developing body image disturbances and eating disorders (Striegel-Moore and Bulik 2007).

\section{Limitations and Future Research Directions}

Several limitations have to be noted about the current study. First, our measure of SVG use relied on users' ratings. Because participants had to estimate the degree of sexualization of the video game characters themselves, these estimations might be biased. However, studies in the area of violent video games demonstrated that user ratings concerning video games are reliable and highly correlated with expert ratings (Busching et al. 2015). Conceptually, although perceived sexualization should be more relevant to understanding its association with users' self-objectification than "objective" sexualization, future studies should examine the extent to which users' perceptions of sexualized content overlap with the degree of sexualization established by independent raters.

Second, we measured SII use by asking participants how frequently they see sexualized images on Instagram. We did not differentiate between the types of persons depicted on these images: whether they are models, celebrities, or peers. There is mixed evidence with regard to whether peer images or celebrity images are more influential on people's body image concerns (Carey et al. 2014; Fardouly and Vartanian 2015). Hence, future studies should include measurements of comparison targets on Instagram.

A third limitation is that we collected data at only two time points, although the model we examined included five steps from media use to body surveillance. To draw definite conclusions, it would be necessary to measure the proposed sequence at five time points. Moreover, we assessed the relevant variables with an interval of 6 months, which is a relatively short period. However, we believe it to be sufficient for the relations that we examined in our study because 
developmental changes happen rapidly in individuals during puberty (Lerner and Steinberg 2009). It would be interesting to see in future studies whether the processes are sustained over several years instead of months. Finally, future research could investigate whether the same model holds for adult users of SVG and SII. Research is still lacking that examines the associations of sexualized media use with body image concerns among women and men beyond early adulthood (Ward 2016).

\section{Practice Implications}

Social media and video games are media formats that adolescents constantly use in their free time (Medienpädagogischer Forschungsverbund Südwest 2019). Therefore, teachers, educators, and also policymakers should be aware of the potential harmful effects of sexualization in these media. Studies suggests that media literacy programs can buffer negative effects of media ideals on female adolescents' thoughts and feelings about their bodies (McLean et al. 2016). Such programs might be implemented at schools to educate adolescents about how sexualized images may contribute to the development of a negative body image. Counselors and educators might also advise adolescents to follow Instagram channels creating body-positive content because initial evidence indicates that body-positive messages improve body image (Cohen et al. 2019). Furthermore, video game developers should be conscious of their responsibility for adolescents' well-being and should create characters in a non-sexualized way.

\section{Conclusion}

The results of our longitudinal study add to the growing body of research demonstrating longitudinal associations of sexualization in the media with adolescents' body image concerns. Our study extends and supports objectification theory by highlighting the role of interactive media like Instagram and video games in this regard and by highlighting appearance comparisons as a meaningful part of the development of adolescents' self-objectification. For future research in this area, we suggest the investigation of possible mediators between muscular-ideal internalization and self-objectification and a more thorough examination of comparison targets on Instagram.

Availability of Data and Material Participants were promised that their data would be used only for scientific purposes. As such, data and material are provided upon request.

Code Availability Mplus code and outputs are provided as Supplementary Material.

Authors' Contributions All authors contributed to the study conception and design. Material preparation and data collection were performed by
Marika Skowronski, analyses were performed by all authors. The first draft of the manuscript was written by Marika Skowronski and all authors commented on previous versions of the manuscript. All authors read and approved the final manuscript.

Funding Information Open Access funding provided by Projekt DEAL. This study was funded by a grant from the Friedrich Ebert Foundation (Friedrich-Ebert-Stiftung) to the first author.

\section{Compliance with Ethical Standards}

We declare that we have no financial or non-financial conflicts of interest. In addition, our participants were provided with informed consent, detailing the risk and benefits of participating in this study. Only human subjects participated in this study.

Open Access This article is licensed under a Creative Commons Attribution 4.0 International License, which permits use, sharing, adaptation, distribution and reproduction in any medium or format, as long as you give appropriate credit to the original author(s) and the source, provide a link to the Creative Commons licence, and indicate if changesweremade. The images or other third partymaterial in this article are included in the article's Creative Commons licence, unless indicated otherwise in a credit line to the material. If material is not included in the article's Creative Commons licence and your intended use is not permitted by statutory regulation or exceeds the permitted use, you will need to obtain permission directly from the copyright holder. To view a copy of this licence, visit http://creativecommons.org/licenses/by/4.0/.

\section{References}

APA Task Force on the Sexualization of Girls. (2007). Report of the APA task force on the sexualization of girls. Washington, DC. Retrieved from http:/www.apa.org/pi/women/programs/girls/report-full.pdf.

Aubrey, J. S. (2006). Effects of sexually objectifying media on selfobjectification and body surveillance in undergraduates: Results of a 2-year panel study. Journal of Communication, 56, 366-386. https://doi.org/10.1111/j.1460-2466.2006.00024.x.

Aubrey, J. S. (2007). The impact of sexually objectifying media exposure on negative body emotions and sexual self-perceptions: Investigating the mediating role of body self-consciousness. Mass Communication and Society, 10, 1-23. https://doi.org/10.1080/ 15205430709337002.

Bozsik, F., Whisenhunt, B. L., Hudson, D. L., Bennett, B., \& Lundgren, J. D. (2018). Thin is in? Think again: The rising importance of muscularity in the thin ideal female body. Sex Roles, 79, 609-615. https://doi.org/10.1007/s11199-017-0886-0.

Busching, R., Gentile, D. A., Krahé, B., Möller, I., Khoo, A., Walsh, D. A., \& Anderson, C. A. (2015). Testing the reliability and validity of different measures of violent video game use in the United States, Singapore, and Germany. Psychology of Popular Media Culture, 4, 97-111. https://doi.org/10.1037/ppm0000004.

Calogero, R. M. (2011). Operationalizing self-objectification: Assessment and related methodological issues. In R. M. Calogero, S. Tantleff-Dunn, \& J. K. Thompson (Eds.), Self-objectification in women: Causes, consequences, and counteractions (pp. 23-49). Washington: American Psychological Association.

Carey, R. N., Donaghue, N., \& Broderick, P. (2014). Body image concern among Australian adolescent girls: The role of body comparisons with models and peers. Body Image, 11, 81-84. https://doi.org/10. 1016/j.bodyim.2013.09.006.

Carrotte, E. R., Prichard, I., \& Lim, M. S. C. (2017). "Fitspiration" on social media: A content analysis of gendered images. Journal of 
Medical Internet Research, 19, e95. https://doi.org/10.2196/jmir. 6368.

Cohen, R., Fardouly, J., Newton-John, T., \& Slater, A. (2019). \#BoPo on Instagram: An experimental investigation of the effects of viewing body positive content on young women's mood and body image. New Media \& Society, 21, 1546-1564. https://doi.org/10.1177/ 1461444819826530.

Cohen, R., Newton-John, T., \& Slater, A. (2017). The relationship between Facebook and Instagram appearance-focused activities and body image concerns in young women. Body Image, 23, 183-187. https://doi.org/10.1016/j.bodyim.2017.10.002.

Cortina, J. M. (1993). What is coefficient alpha? An examination of theory and applications. The Journal of Applied Psychology, 78, 98-104. https://doi.org/10.1037/0021-9010.78.1.98.

DAK-Gesundheit. (2017). WhatsApp, Instagram und co. - so süchtig macht Social Media. Retrieved from https://www.eukidsonline.de/ wp-content/uploads/DAK-Studie-Social-Media-Abhängigkeit.pdf.

Davis, S. E. (2018). Objectification, sexualization, and misrepresentation: Social media and the college experience. Social Media + Society, 4, 205630511878672. https://doi.org/10.1177/2056305118786727.

Deighton-Smith, N., \& Bell, B. T. (2018). Objectifying fitness: A content and thematic analysis of \#fitspiration images on social media. Psychology of Popular Media Culture, 7, 467-483. https://doi.org/ $10.1037 / \mathrm{ppm} 0000143$

Dill, K. E., \& Thill, K. P. (2007). Video game characters and the socialization of gender roles: Young people's perceptions mirror sexist media depictions. Sex Roles, 57, 851-864. https://doi.org/10.1007/ s11199-007-9278-1.

Downs, E., \& Smith, S. L. (2010). Keeping abreast of hypersexuality: A video game character content analysis. Sex Roles, 62, 721-733. https://doi.org/10.1007/s11199-009-9637-1.

Enders, C., \& Bandalos, D. (2001). The relative performance of full information maximum likelihood estimation for missing data in structural equation models. Structural Equation Modeling: A Multidisciplinary Journal, 8, 430-457. https://doi.org/10.1207/ S15328007SEM0803 5.

Fardouly, J., Diedrichs, P. C., Vartanian, L. R., \& Halliwell, E. (2015). The mediating role of appearance comparisons in the relationship between media usage and self-objectification in young women. Psychology of Women Quarterly, 39, 447-457. https://doi.org/10. $1177 / 0361684315581841$.

Fardouly, J., \& Vartanian, L. R. (2015). Negative comparisons about one's appearance mediate the relationship between Facebook usage and body image concerns. Body Image, 12, 82-88. https://doi.org/ 10.1016/j.bodyim.2014.10.004.

Fardouly, J., Willburger, B. K., \& Vartanian, L. R. (2017). Instagram use and young women's body image concerns and self-objectification: Testing mediational pathways. New Media \& Society, 20, 1380 1395. https://doi.org/10.1177/1461444817694499.

Fatt, S. J., Fardouly, J., \& Rapee, R. M. (2019). \#malefitspo: Links between viewing fitspiration posts, muscular-ideal internalisation, appearance comparisons, body satisfaction, and exercise motivation in men. New Media \& Society, 21, 1311-1325. https://doi.org/10. $1177 / 1461444818821064$.

Feltman, C. E., \& Szymanski, D. M. (2018). Instagram use and selfobjectification: The roles of internalization, comparison, appearance commentary, and feminism. Sex Roles, 78, 311-324. https://doi.org/ 10.1007/s11199-017-0796-1.

Fox, J., Ralston, R. A., Cooper, C. K., \& Jones, K. A. (2015). Sexualized avatars lead to women's self-objectification and acceptance of rape myths. Psychology of Women Quarterly, 39, 349-362. https://doi. org/10.1177/0361684314553578.

Fredrickson, B. L., \& Roberts, T.-A. (1997). Objectification theory: Toward understanding women's lived experiences and mental health risks. Psychology of Women Quarterly, 21, 173-206. https://doi.org/ 10.1111/j.1471-6402.1997.tb00108.x.
Ghaznavi, J., \& Taylor, L. D. (2015). Bones, body parts, and sex appeal: An analysis of \#thinspiration images on popular social media. Body Image, 14, 54-61. https://doi.org/10.1016/j.bodyim.2015.03.006.

Hair, J. F., Black, W. C., Babin, B. J., \& Anderson, R. E. (2019). Multivariate data analysis (Eighth edition). Andover: Cengage Learning EMEA.

Harrison, K., \& Bond, B. J. (2007). Gaming magazines and the drive for muscularity in preadolescent boys: A longitudinal examination. Body Image, 4, 269-277. https://doi.org/10.1016/j.bodyim.2007. 03.003.

Hayes, A. F., \& Scharkow, M. (2013). The relative trustworthiness of inferential tests of the indirect effect in statistical mediation analysis: Does method really matter? Psychological Science, 24, 1918-1927. https://doi.org/10.1177/0956797613480187.

Helfert, S., \& Warschburger, P. (2013). The face of appearance-related social pressure: Gender, age and body mass variations in peer and parental pressure during adolescence. Child and Adolescent Psychiatry and Mental Health, 7, 1-11. https://doi.org/10.1186/ 1753-2000-7-16

Holland, G., \& Tiggemann, M. (2016). A systematic review of the impact of the use of social networking sites on body image and disordered eating outcomes. Body Image, 17, 100-110. https://doi.org/10.1016/ j.bodyim.2016.02.008.

Hopper. (2019). Instagram rich list 2019. Retrieved from https://www. hopperhq.com/blog/instagram-rich-list/.

Hu, L.-T., \& Bentler, P. M. (1998). Fit indices in covariance structure modeling: Sensitivity to underparameterized model misspecification. Psychological Methods, 3, 424-453. https://doi. org/10.1037/1082-989X.3.4.424.

Hussey, I., \& Hughes, S. (2020). Hidden invalidity among 15 commonly used measures in social and personality psychology. Advances in Methods and Practices in Psychological Science, 3, 166-184. https://doi.org/10.1177/2515245919882903.

Karsay, K., Knoll, J., \& Matthes, J. (2017). Sexualizing media use and self-objectification: A meta-analysis. Psychology of Women Quarterly, advance online publication, 42, 9-28. https://doi.org/10. $1177 / 0361684317743019$.

Keery, H., van den Berg, P., \& Thompson, J. K. (2004). An evaluation of the tripartite influence model of body dissatisfaction and eating disturbance with adolescent girls. Body Image, 1, 237-251. https://doi. org/10.1016/j.bodyim.2004.03.001.

Knauss, C., Paxton, S. J., \& Alsaker, F. D. (2008). Body dissatisfaction in adolescent boys and girls: Objectified body consciousness, internalization of the media body ideal and perceived pressure from media. Sex Roles, 59, 633-643. https://doi.org/10.1007/s11199-008-94747.

Lerner, R. M., \& Steinberg, L. (2009). Handbook of adolescent psychology. Hoboken: John Wiley \& Sons, Inc.

Little, T. D., Rhemtulla, M., Gibson, K., \& Schoemann, A. M. (2013). Why the items versus parcels controversy needn't be one. Psychological Methods, 18, 285-300. https://doi.org/10.1037/ a0033266.

Liu, R., \& Suh, A. (2017). Self-branding on social media: An analysis of style bloggers on Instagram. Procedia Computer Science, 124, 12 20. https://doi.org/10.1016/j.procs.2017.12.124.

Lynch, T., Tompkins, J. E., van Driel, I. I., \& Fritz, N. (2016). Sexy, strong, and secondary: A content analysis of female characters in video games across 31 years. Journal of Communication, 66, 564 584. https://doi.org/10.1111/jcom.12237.

Martins, Y., Tiggemann, M., \& Kirkbride, A. (2007). Those speedos become them: The role of self-objectification in gay and heterosexual men's body image. Personality \& Social Psychology Bulletin, 33, 634-647. https://doi.org/10.1177/0146167206297403.

McKinley, N. M., \& Hyde, J. S. (1996). The Objectified Body Consciousness Scale: Development and validation. Psychology of 
Women Quarterly, 20, 181-215. https://doi.org/10.1111/j.14716402.1996.tb00467.x.

McLean, S. A., Paxton, S. J., \& Wertheim, E. H. (2016). Does media literacy mitigate risk for reduced body satisfaction following exposure to thin-ideal media? Journal of Youth and Adolescence, 45, 1678-1695. https://doi.org/10.1007/s10964-016-0440-3.

Medienpädagogischer Forschungsverbund Südwest. (2019). JIM-Studie 2019: Jugend, Information, Medien. Retrieved from https://www. mpfs.de/studien/jim-studie/2019/.

Moradi, B. (2010). Addressing gender and cultural diversity in body image: Objectification theory as a framework for integrating theories and grounding research. Sex Roles, 63, 138-148. https://doi.org/10. 1007/s11199-010-9824-0.

Moradi, B. (2011). Objectification theory: Areas of promise and refinement. The Counseling Psychologist, 39, 153-163. https://doi.org/10. 1177/0011000010384279.

Moradi, B., \& Huang, Y.-P. (2008). Objectification theory and psychology of women: A decade of advances and future directions. Psychology of Women Quarterly, 32, 377-398. https://doi.org/10. 1111/j.1471-6402.2008.00452.x.

Morry, M. M., \& Staska, S. L. (2001). Magazine exposure: Internalization, self-objectification, eating attitudes, and body satisfaction in male and female university students. Canadian Journal of Behavioural Science / Revue Canadienne des Sciences du Comportement, 33, 269-279. https://doi.org/10.1037/h0087148.

Noll, S. M., \& Fredrickson, B. L. (1998). A mediational model linking self-objectification, body shame, and disordered eating. Psychology of Women Quarterly, 22, 623-636. https://doi.org/10.1111/j.14716402.1998.tb00181.x.

Peat, C. M., \& Muehlenkamp, J. J. (2011). Self-objectification, disordered eating, and depression: A test of mediational pathways. Psychology of Women Quarterly, 35, 441-450. https://doi.org/10. $1177 / 0361684311400389$.

Raftery, A. E. (1995). Bayesian model selection in social research. Sociological Methodology, 25, 111-163. https://doi.org/10.2307/ 271063.

Read, G. L., Lynch, T., \& Matthews, N. L. (2018). Increased cognitive load during video game play reduces rape myth acceptance and hostile sexism after exposure to sexualized female avatars. Sex Roles, 5, 279-698. https://doi.org/10.1007/s11199-018-0905-9.

Robinson, L., Prichard, I., Nikolaidis, A., Drummond, C., Drummond, M., \& Tiggemann, M. (2017). Idealised media images: The effect of fitspiration imagery on body satisfaction and exercise behaviour. Body Image, 22, 65-71. https://doi.org/10.1016/j.bodyim.2017.06. 001 .

Saiphoo, A. N., \& Vahedi, Z. (2019). A meta-analytic review of the relationship between social media use and body image disturbance. Computers in Human Behavior, 101, 259-275. https://doi.org/10. 1016/j.chb.2019.07.028.

Schaefer, L. M., Harriger, J. A., Heinberg, L. J., Soderberg, T., \& Kevin Thompson, J. (2017). Development and validation of the Sociocultural Attitudes Towards Appearance Questionnaire-4Revised (SATAQ-4R). The International Journal of Eating Disorders, 50, 104-117. https://doi.org/10.1002/eat.22590.

Schermelleh-Engel, K., Moosbrugger, H., \& Müller, H. (2003). Evaluating the fit of structural equation models: Tests of significance and descriptive goodness-of-fit measures. Methods of Psychological Research Online, 8(2), 23-74.

Schlomer, G. L., Bauman, S., \& Card, N. A. (2010). Best practices for missing data management in counseling psychology. Journal of Counseling Psychology, 57, 1-10. https://doi.org/10.1037/ a0018082.

Sevic, S., Ciprić, A., Buško, V., \& Štulhofer, A. (2019). The relationship between the use of social networking sites and sexually explicit material, the internalization of appearance ideals and body self-surveillance: Results from a longitudinal study of male adolescents.
Journal of Youth and Adolescence, 49, 383-398. https://doi.org/ 10.1007/s10964-019-01172-2.

Shroff, H., \& Thompson, J. K. (2006). The tripartite influence model of body image and eating disturbance: A replication with adolescent girls. Body Image, 3, 17-23. https://doi.org/10.1016/j.bodyim.2005. 10.004 .

Striegel-Moore, R. H., \& Bulik, C. M. (2007). Risk factors for eating disorders. The American Psychologist, 62, 181-198. https://doi. org/10.1037/0003-066X.62.3.181.

Thompson, J. K., Heinberg, L. J., Altabe, M., \& Tantleff-Dunn, S. (1999). Exacting beauty: Theory, assessment, and treatment of body image disturbance. Washington: American Psychological Association.

Thompson, J. K., van den Berg, P., Roehrig, M., Guarda, A. S., \& Heinberg, L. J. (2004). The Sociocultural Attitudes Towards Appearance Scale-3 (SATAQ-3): Development and validation. The International Journal of Eating Disorders, 35, 293-304. https://doi.org/10.1002/eat.10257.

Thompson, J. K., Heinberg, L., \& Tantleff-Dunn, S. (1991). The Physical Appearance Comparison Scale. The Behavior Therapist, 14, 174.

Tiggemann, M., \& Lynch, J. E. (2001). Body image across the life span in adult women: The role of self-objectification. Developmental Psychology, 37, 243-253. https://doi.org/10.1037/0012-1649.37.2. 243.

Tiggemann, M., \& Williams, E. (2011). The role of self-objectification in disordered eating, depressed mood, and sexual functioning among women. Psychology of Women Quarterly, 36, 66-75. https://doi. org $/ 10.1177 / 0361684311420250$.

Tiggemann, M., \& Zaccardo, M. (2015). "Exercise to be fit, not skinny": The effect of fitspiration imagery on women's body image. Body Image, 15, 61-67. https://doi.org/10.1016/j.bodyim.2015.06.003.

Tiggemann, M., \& Zaccardo, M. (2018). 'Strong is the new skinny': A content analysis of \#fitspiration images on Instagram. Journal of Health Psychology, 23, 1003-1011. https://doi.org/10.1177/ 1359105316639436.

Twenge, J. M., Martin, G. N., \& Spitzberg, B. H. (2019). Trends in U.S. adolescents' media use, 1976-2016: The rise of digital media, the decline of TV, and the (near) demise of print. Psychology of Popular Media Culture, 8, 329-345. https://doi.org/10.1037/ppm0000203.

van de Schoot, R., Lugtig, P., \& Hox, J. (2012). A checklist for testing measurement invariance. European Journal of Developmental Psychology, 9, 486-492. https://doi.org/10.1080/17405629.2012. 686740.

van den Berg, P., Thompson, J. K., Obremski-Brandon, K., \& Coovert, M. (2002). The tripartite influence model of body image and eating disturbance. Journal of Psychosomatic Research, 53, 1007-1020. https://doi.org/10.1016/S0022-3999(02)00499-3.

Vandenberg, R. J., \& Lance, C. E. (2000). A review and synthesis of the measurement invariance literature: Suggestions, practices, and recommendations for organizational research. Organizational Research Methods, 3, 4-70. https://doi.org/10.1177/ 109442810031002.

Vandenbosch, L., Driesmans, K., Trekels, J., \& Eggermont, S. (2017). Sexualized video game avatars and self-objectification in adolescents: The role of gender congruency and activation frequency. Media Psychology, 20, 221-239. https://doi.org/10.1080/ 15213269.2016.1142380.

Vandenbosch, L., \& Eggermont, S. (2012). Understanding sexual objectification: A comprehensive approach toward media exposure and girls' internalization of beauty ideals, self-objectification, and body surveillance. Journal of Communication, 62, 869-887. https://doi. org/10.1111/j.1460-2466.2012.01667.x.

Vandenbosch, L., \& Eggermont, S. (2015). The role of mass media in adolescents' sexual behaviors: Exploring the explanatory value of the three-step self-objectification process. Archives of Sexual Behavior, 44, 729-742. https://doi.org/10.1007/s10508-014-02924. 
Vandenbosch, L., \& Eggermont, S. (2016). The interrelated roles of mass media and social media in adolescents' development of an objectified self-concept. Communication Research, 43, 1116-1140. https:// doi.org/10.1177/0093650215600488.

de Vries, D. A., Peter, J., de Graaf, H., \& Nikken, P. (2016). Adolescents' social network site use, peer appearance-related feedback, and body dissatisfaction: Testing a mediation model. Journal of Youth and Adolescence, 45, 211-224. https://doi.org/10.1007/s10964-0150266-4.

Wang, Y., Xie, X., Fardouly, J., Vartanian, L. R., \& Lei, L. (2019). The longitudinal and reciprocal relationships between selfie-related behaviors and self-objectification and appearance concerns among adolescents. New Media \& Society, 9, 1-22. https://doi.org/10.1177/ 1461444819894346.

Ward, L. M. (2016). Media and sexualization: State of empirical research, 1995-2015. Journal of Sex Research, 53, 560-577. https://doi.org/ 10.1080/00224499.2016.1142496.

Publisher's Note Springer Nature remains neutral with regard to jurisdictional claims in published maps and institutional affiliations. 